\title{
A REVIEW OF THE DYNAMICS OF GLASSICAL TRIPLE STARS
}

(Invited Paper)

\section{R. S. HARRINGTON}

U.S. Naval Observatory, Washington, D.C.

\begin{abstract}
RESUMEN
Someramente se bosquejan cuatro maneras de estudiar la dinámica de sistemas triples, a saber: procedimiento analítico, cualitativo, numérico y observacional. Una discusión de la estabilidad de triples clásicas (con rotación y jerarquía) basada en la aplicación del método de von Zeipel al problema general de tres cuerpos y en experimentación numérica, resume el resultado de los avances analíticos.

Finalmente, se da un resumen de investigaciones recientes relativas a la dinámica de triples que no sean sistemas en revolución.
\end{abstract}

\begin{abstract}
Four ways of studying the dynamics of triple systems are outlined, namely the analytical, the qualitative, the numerical, and the observational approach. A discussion of the stability of classical (revolutional, hierarchical) triples summarizes the results of analytical developments, based on the application of von Zeipel's method to the general three-body problem, and of numerical experimentation. Finally, a summary is given of recent investigations concerning the dynamics of triples other than the revolutional systems.
\end{abstract}

The study of the dynamics of triple stars requires the solution of one of the classic problems of celestial mechanics - the general three-body problem. That is, triple stars consist of three bodies, of arbitrary masses, with arbitrary spacing, which generally can be considered as point gravitational sources; it is the motion in this kind of system that we want to determine. Note that, since we are considering point masses (spherical symmetry), there is a lower limit on separations, of approximately $10-20$ stellar radii. Also, the effects of field stars and the general galactic field are neglected, which imposes an upper limit of perhaps 100000 astronomical units on the separations.

There are several ways available to us to learn something about such a problem. First, there is the analytical approach. In this approach, we set up the differential equations governing the motions in , the system and attempt to find the solution to the equations. Often, restrictive assumptions are imposed, and the solutions are in the form of infinite series rather than being closed. For the general threebody problem, there are no such solutions available.

Second, there is the qualitative approach. This approach also attacks the equations of motion analytically, but, rather than searching for formal solutions, only general properties, such as conditions for escape, limits to the motion, and so forth, are obtained. This is an approach Szebehely (e.g., 1974), among others, has used very successfully.

A third approach is the numerical one. Here we use a computer to solve the equations of motion numerically for a given system with specified masses and initial conditions. This approach is very popular now, with the advent of large, high-speed machines, and it allows us to consider systems that are intractable analytically. However, because we can only integrate one system at a time, and that 
for only a finite time interval, it is very difficult to establish, with confidence, any general properties of the motion valid for all time. This is an approach used by many investigators, some of whom are reporting later in these proceedings. Besides the limitations of finite time intervals, there are problems of truncation and round-off errors that affect the solutions and may make them increasingly unrealistic with time.

A fourth approach is observational. This is a point often over-looked by the theoretician, but there is much dynamical information available just by looking up. As should by now be quite apparent, the universe is full of triple stars; hence there must be solutions of the three-body problem that are stable against dynamical decay. (Of course no system has been observed to time equals infinity, but $I$ am willing to take $10^{10}$ years as a first approximation). However, virtually all of the triple stars that are not, for other reasons, thought to be young systems are arranged in a hierarchical pattem. That is, two of the stars are close together compared to the distance of either from the third, and this should tell us something about the region in phase-space that contains stable systems. These systems, containing a close binary and an always distant tertiary, are the "classical" triple stars, the kind with which we are concerned here.

Unfortunately, there has not been much work done on the dynamics of these systems, mostly because there is very little to do. To a first approximation the motion can be decoupled into 2-body motion in the binary, plus 2-body motion of the binary barycenter and the tertiary. Most of the numerical work mentioned earlier, on the other hand, has been directed toward studies of dynamically decaying systems or toward finding periodic orbits in the general case. Neither type of configuration qualifies as "classical", and indeed being classical is an indication of being uninteresting. In addition, from an obervational point of view, the perturbative effects of the 3-body motion are generally undetectable and do not need to be considered in the orbit computation. Indeed, there are only two cases known so far -Xi Ursae Majoris (Heintz 1967) and Zeta Aquarii (Harrington 1968) - in which the 3-body effects have even been observed at all.

What more can be said about the dynamics of classical triples? Fortunately, because these systems are arranged in a hierarchical pattern, an analytic development is possible. Because the distance separating the components of the binary is small compared to the distance of the binary-barycenter from the tertiary, one of the canonical transformation/averaging techniques, such as that due to von Zeipel, can be applied. In this procedure, the force function in the equations of motion for the system is expanded in a power series in the small parameter (in this case, the distance ratio). For application, the series can be truncated at some desired power of the small parameter; carrying the series to higher power produces greater accuracy but also considerably more work in the analysis. A canonical transformation is sought that (a) identifies certain additional constants (integrals) of the motion, at the same time eliminating certain angular variables, and (b) produces expressions for the elements as functions of time, these expressions also being power series in the small parameter.

In the stellar problem, the distinguishing characteristic is that the masses have to be considered as comparable. Because the small parameter is the distance ratio, a feature in common with the lunar theory, early developments attempted to generalize that theory to the non-small-mass case. However, the lunar theory assumes small eccentricities and inclinations, clearly not the case for multiple stars, so a fresh start would seem appropriate.

Performing the required von Zeipel expansion for the general 3-body problem (see Harrington 1969 for a complete discussion), the first term produces the 2-body motion of the binary, the second term the 2-body motion of the tertiary and binary barycenter, and the remaining terms the perturbations from the various 2-body motions. For remaining developments, only the third term has been carried through the entire procedure analytically, while the fourth term has been treated numerically.

When the above procedure is carried out, several significant results emerge. First, both semi-major axes can be expressed as a constant (an integral of the motion) plus purely periodic functions of time, with the coefficients of the periodic functions containing at least the first power of the small parameter. That is, the semi-major axes have only small periodic variations- no secular variation. This in. 
turn means a classical triple, by itself, has always been, and will always remain, a classical triple; it can neither break-up by dynamical decay nor could have envolved from a trapezium system without some additional external influence. This is what makes a classical system fundamentally different from a trapezium system - dynamical decay has terminated.

Second, if only the first perturbative term is consicered, the eccentricity of the tertiary's orbit is also a constant plus small periodic variations. However, the eccentricity of the binary and the mutual inclination of the two orbits show large, coupled variations, such that the eccentricity increases as the inclination decreases. The most extreme case is when the two orbits are perpendicular most of the time; then, because periodically the inclination rapidly approaches zero and the binary eccentricity approaches unity, the periastron separation in the binary approaches someting comparable to typical stellar diameters, and the binary experiences a very close approach if not a collision. In any case the binary would be violently disrupted, so systems with a mutual inclination just over $90^{\circ}$ are unstable.

Third, if the second perturbative term is included, the tertiary eccentricity is no longer constant on average. Indeed, it can be shown numerically that there is no other equivalent constant, and thus there is no other isolating integral of the motion. Since this additional isolating integral is necessary if the motion is ever to be expressed completely in analytical terms, we know that the dynamics of even classical triples can not be described, in complete generality, entirely analytically.

There is a limitation to the above procedure, a limitation imposed by the question of convergence. That is, there is the question of the limit on the small parameter (the distance ratio) that will make this process work. This is equivalent to asking what the limit on the small parameter might be to insure stability of the system, i.e., that it is truly a classical triple.

The best way to approach this problem is by numerical experimentation. A large number of 3-body systems, with masses and initial conditions suitably chosen, have been numerically integrated, and the semi-major axes and eccentricities monitored to es- tablish stability (Harrington 1975). By suitable variations of initial elements from one system to the next, the parameters pertinent to stability, and their limiting values, can be determined. It has been found that the most significant parameter is the ratio of the semi-major axis of the binary orbit $\left(a_{1}\right)$ to the periastron distance of the tertiary $\left(q_{2}\right)$. This is not surprising, since the major perturbation from the tertiary comes at closest approach, while the relatively high orbital frequency of the binary makes a measure of the average separation more meaningful. Further, there is a bifurcation into two classes -the co-revolving and counter-revolving systems - with the separation coming at the perpendicular instability. Within each class the limiting value of $a_{1} / q_{2}$ is constant and does not depend on the relative inclination of the orbits. The dependence on the masses is relatively weak for the mass ratios normally encountered in triple stars. Quantitatively, the following sufficient condition for stability has been suggested:

$$
\begin{aligned}
\mathrm{a}_{1} / \mathrm{q}_{2} & <\mathrm{K} \log (3 / 2) / \log \left[\frac{\mathrm{m}_{1}+\mathrm{m}_{2}+\mathrm{m}_{3}}{\mathrm{~m}_{1}+\mathrm{m}_{2}}\right] \\
& =\mathrm{K} \log (3 / 2) / \log \left[1+\frac{\mathrm{m}_{3}}{\mathrm{~m}_{1}+\mathrm{m}_{2}}\right]
\end{aligned}
$$

The masses of the components of the binary are $m_{1}$ and $m_{2}$; that of the tertiary is $m_{3}$. For corevolving systems, $\mathrm{K}$ is approximately 0.28 , while for counter-revolving systems it is approximately 0.36 . For all observed triples for which $a_{1} / q_{2}$ is known, it is less than 0.2 , consistent with the assumption that the observed classical triples are stable. Note that the above is an average upper limit, established by a random statistical sampling of possible initial conditions. It is possible to have stable systems with larger values of $a_{1} / q_{2}$ (e.g., in periodic orbits), but the probability of finding such is probably small.

Until recently it has been assumed that all triples observed to be in a hierarchical arrangement were classical triples, and that the only stable stellar configuration was a hierarchical one. Neither of these assumptions need be true. First, consider the observed hierarchical systems. Szebehely (1971) has listed 6 types of motion possible in a 3-body system; the revolutional type is the one that we have been considering here. There are two stages of motion 
in unstable systems prior to escape, known as interplay and ejection. In interplay, all separations are of the same order, motions are rapid, and perturbations are strong. In ejection, one component has left the other two in a very extended orbit, but without positive energy, so it must eventually return to the pair. These ejection orbits may be very extended, however, and therefore have very long periods. Further, most of the time the tertiary is on the more remote parts of the ejection orbit, making the system appear to be classical if the elements are not known. In addition, the system can go through many cycles of interplay and ejection, with different components ejecting at different times, giving very extensive life-times for such apparently stable systems. Numerical experiments have shown, for instance, that if the separations are of the order of $10^{3} \mathrm{AU}$, the time to decay can be as large as $10^{\circ}$ years.

Because of time reversibility, capture of a single star by a binary is one way to lead to motion as has just been described. The probability of capture is small, but it is not zero, since the binary can at least temporarily absorb energy from the field star. We know, however, from the discussion of the lack of secular terms in the semi-major axes, that capture can not lead to a stable classical system. Therefore, there must be sequences of ejection and interplay leading ultimately to escape of one component. However, as before, the escaper need not be the one originally captured; that is, the remaining binary may consist of two stars that previously had lead astrophysically independent lives. This could be a possibility for something like the Sirius system, where the less massive star is apparently older, but the separation is too great to permit mass exchange.

As for other modes of stability, much work has been done very recently on establishing periodic orbits in the general 3-body problem. Many such orbits have been found, and they are not all of the classical type. Further, some of these appear to be stable, and again they spend some of their lifetime in an apparently hierarchical configuration. While the probability of achieving such an orbit is small, it is not zero. Thus, there exists the possibility that at least a few of the systems that have been classified as classical on appearances alone are either unstable, or stable but non-classical.

Something should be said about systems of higher multiplicity. As with triples, all presumably old systems are arranged in hierarchies of spacings, though, as the number of components increases, the permutations and combinations increase drastically. The motions in such systems can be represented in the first approximation by various combinations of 2-body motions, with the effects of other bodies again being added as small perturbations. Except under some very restrictive approximations, there has been no work done on these higher-order systems, though an extension of the 3-body results in various combinations would probably generate the next order of solution. Again there is the question of stability, which has not been answered for these systems, but the presence of large numbers of high multiplicity systems with old components indicates there must be at least some degree of stability, even if it is not complete.

\section{REFERENCES}

Harrington, R. S. 1968 A J., 73, 508.

Harrington, R. S. 1969, Celes. Mech., 1, 200.

Harrington, R. S. 1975, A. J., 80, 1081.

Heintz, W. 1967, Astr. Nachr., 289, 269.

Szebehely, V. 1971, Celes. Mech., 4, 116.

Szebehely, V. 1974, Celes. Mech., 9, 359.

\section{DISCUSSION}

Aarseth: How did you determine the logarithmic mass factor in the stability criterion? Harrington: Empirically. This was a fit to stability results from numerical integration of triple systems with only different values of the masses. This fit is not perfect and should only be considered a sufficient condition for stability.

Ovenden: The numerical statistical approach which you have discussed so clearly is a powerful approach to the multiple-star dynamical problem. However, a word of caution is necessary. All statements of probability require a definition of prior probability-in your case, perhaps, that all combinations of initial parameters are equally possible. But Nature 
knows nothing of our prejudices, and the systems we find in Nature will be, according to our probability criterion, highly improbable. Indced, it is precisely the improbability of systems found in Nature that gives us information about their origin. Has your choice of initial conditions been determined by astrophysical considerations?

Harrington: Yes, initial conditions are taken from distributions suggested by observed frequency distribution.

Evans: I think this expresses the difference in our point of view. I was looking at the average parameters in observed systems, and you are looking at the limits theoretically attainable.

Harrington: For other reasons, I suspect the limit to multiplicity may be closer to what you suggested earlicr than what is deduced here. However, my limit may indicate there are one or two systems of very high multiplicity.

Harrington: I would like to add that these small values for limits of distance ratios that were discussed here are the basis for my questioning the input values David Evans used in his review to estimate the maximum possible number of components in a stable multiple system. Hence, we have concluded a little wager on the subject. He is betting $\$ 5.00$ that no one will find a hierarchical multiple system with more than 10 components in the next 10 years, and I am betting a like amount that someone will. 